\title{
On the Closed-Loop Structure of $H^{\infty}$ Control Systems
}

\author{
Shun UshidA*, Toshitomo OHBA*, \\ Hidenori KIMURA* and Yasuaki OISHI*
}

\begin{abstract}
In this paper, we show that the order of the closed-loop $H^{\infty}$ control systems using central controllers is determined by the sum of the numbers of stable invariant zeros of $P_{12}(s)$ and $P_{21}(s)$. This fact gives a sharp contrast with the LQG case where the order of the closed-loop system is always identical to that of the plant. Furthermore, using this result, we derive a new explicit form of the closed-loop transfer function of $H^{\infty}$ control systems based on the chain-scattering approach, which clarifies the fundamental structure of $H^{\infty}$ control systems.
\end{abstract}

Key Words: $H^{\infty}$ control, chain-scattering approach, closed-loop system, McMillan degree, invariant zero

\section{Introduction}

In the 1980's, the standard $H^{\infty}$ control problem has been studied by many researchers $\left.\left.\left.\left.{ }^{1) \sim 4}\right), 16\right), 17\right), 19\right)$. As a result, various methods and algorithms for constructing $H^{\infty}$ controller have been established and $H^{\infty}$ control has become a powerful tool for robust control. Actually, inexpensive software packages for computing $H^{\infty}$ controllers are now commercially available for most users and they enáble us to easily apply $H^{\infty}$ control to real control systems using them. Furthermore, in order to deal with more complicated systems, a variety of $H^{\infty}$ control techniques has been developed in the last decade ${ }^{5) \sim 10)}$.

It is questionable, however, that nothing serious remains to be solved. In particular, the closed-loop structure of $H^{\infty}$ control systems are still to be exploited, according to our opinion. Our investigation in this paper will allow us to enhance the understanding of $H^{\infty}$ control theory, which is quite rich in a logical structure itself. Such a theoretical background of $H^{\infty}$ control will give us a deep insight and be helpful to deal with the application for more complicated systems.

In LQG control ${ }^{12) ~ 15)}$, or in any other control scheme that uses quasi state feedback, i.e., state feedback with an observer, the order of a closed-loop system is identical to that of a plant. The insertion of a controller does not increase the intrinsic complexity of a control system. This is a remarkable property of modern control methods. In $H^{\infty}$ control, however, the situation is different. The order of a closed-loop system is no longer identical to that of a plant. Instead, the McMillan degree of a closed system is determined by a different factor. In the so-called one-block case, i.e., if both $P_{12}$ and $P_{21}$ are square but

* Department of Mathematical Engineering and Information Physics, Graduate School of Engineering, the University of Tokyo, Tokyo, 113-8656, JAPAN

(Received March 15, 2000)

(Revised October 5, 2000) not necessarily of the same size, this problem has been fully analyzed based on the classical interpolation theory ${ }^{16)}{ }^{17)}$. Furthermore, it has been pointed out that all stable invariant zeros of $P_{12}$ and $P_{21}$ are hidden modes of the generalized $H^{\infty}$ control systems ${ }^{6}$ ).

In this paper, we derive an explicit form of the closedloop transfer function of an $H^{\infty}$ control system, which has not been derived so far within the knowledge of the authors $^{(1)}$. This derivation is carried out in the chainscattering framework ${ }^{11)}$. There, the solvability of $H^{\infty}$ control problem is reduced to the existence of $J$-lossless factorization for the chain-scattering form of a plant. Our result is derived by calculating the $J$-lossless factor directly, which is discussed in Section 3 in detail. Based on our representation of a closed-loop system, we prove the fact pointed out by Liu and Mita ${ }^{6)}$ in a more explicit way. Our result, which holds for a four-block case, too, is a generalization of the results by Limebeer et al. ${ }^{16), 17)}$. As a consequence of this result, we shall also provide a new interpretation of maximum augmentation, which is an important tool in the chain-scattering approach ${ }^{11)}$.

Notation: We write the transfer function derived from a state-space representation as

$$
\left[\begin{array}{l|l}
A & B \\
\hline C & D
\end{array}\right]:=D+C(s I-A)^{-1} B
$$

We write $H^{\infty}$ norm, a maximum singular value and a maximum eigenvalue as $\|\cdot\|_{\infty}, \bar{\sigma}\{\cdot\}$ and $\lambda_{\max }(\cdot)$, respectively. We denote a set of all rational stable proper matrix whose norm is less than 1 as $\boldsymbol{B} \boldsymbol{H}^{\infty}$. The homographic transformation is given by

$$
H M\left(\left[\begin{array}{ll}
\Theta_{11} & \Theta_{12} \\
\Theta_{21} & \Theta_{22}
\end{array}\right] ; S\right):=\left(\Theta_{11} S+\Theta_{12}\right)\left(\Theta_{21} S+\Theta_{22}\right)^{-1}
$$

This transformation has the following cascade property, which is one of advantages to use the chainscattering approach rather than the LFT approach to

(1) Our previous paper ${ }^{18)}$ contains incorrect result. This paper is the modified version and also provides some new results. 
$H^{\infty}$ control. For two systems $\Theta_{1}$ and $\Theta_{2}$, there holds $H M\left(\Theta_{1} ; H M\left(\Theta_{2} ; S\right)\right)=H M\left(\Theta_{1} \Theta_{2} ; S\right)$.

\section{Preliminaries}

Consider the plant

$$
\left[\begin{array}{l}
z \\
y
\end{array}\right]=P(s)\left[\begin{array}{l}
w \\
u
\end{array}\right]
$$

where $z$ is a control output $(\operatorname{dim}(z)=m), y$ is an observation output $(\operatorname{dim}(y)=q), w$ is an exogenous input $(\operatorname{dim}(w)=r)$ and $u$ is a control input $(\operatorname{dim}(u)=p)$, respectively. The state-space form of the plant is represented as

$$
P(s)=\left[\begin{array}{c|cc}
A & B_{1} & B_{2} \\
\hline C_{1} & D_{11} & D_{12} \\
C_{2} & D_{21} & 0
\end{array}\right]=\left[\begin{array}{cc}
P_{11}(s) & P_{12}(s) \\
P_{21}(s) & P_{22}(s)
\end{array}\right]
$$

Consider the controller $u=K(s) y$. Let $\Phi(s)$ be the closed-loop transfer function from $w$ to $z$ (Fig. 1), which is given by

$$
\Phi(s)=P_{11}(s)+P_{12}(s) K(s)\left(I-P_{22}(s) K(s)\right)^{-1} P_{21}(s) .
$$

Fig. 1 A closed-loop system

The $H^{\infty}$ control problem is to find all controllers $K(s)$ that satisfy. $\|\Phi(s)\|_{\infty}<\gamma$ and internally stabilize the closed-loop system. A solvability condition has been obtained by various methods $\left.\left.{ }^{11)}, 19\right), 20\right)$. In this paper, we take the chain-scattering approach ${ }^{11)}$ to investigate the closed-loop structure of $H^{\infty}$ control systems.

We make the following assumptions.

(A1) $\left(A, B_{2}\right)$ is stabilizable and $\left(C_{2}, A\right)$ is detectable.

(A2) $P_{12}(s)$ and $P_{21}(s)$ have no invariant zeros on the $j \omega$-axis.

Before stating the solvability condition of the $H^{\infty}$ control problem, we introduce some notation which is used throughout this paper:

$$
\begin{gathered}
B:=\left[B_{1} B_{2}\right], C_{z}:=\left[\begin{array}{c}
C_{1} \\
0
\end{array}\right], D_{z}:=\left[\begin{array}{cc}
D_{11} & D_{12} \\
I_{r} & 0
\end{array}\right], \\
C:=\left[\begin{array}{c}
C_{1} \\
C_{2}
\end{array}\right], B_{w}:=\left[\begin{array}{ll}
B_{1} & 0
\end{array}\right], D_{w}:=\left[\begin{array}{cc}
D_{11} & I_{m} \\
D_{21} & 0
\end{array}\right], \\
J_{\gamma}:=\left[\begin{array}{cc}
I_{m} & 0 \\
0 & -\gamma^{2} I_{r}
\end{array}\right], \quad J_{\gamma}^{\prime}:=\left[\begin{array}{cc}
I_{r} & 0 \\
0 & -\gamma^{2} I_{m}
\end{array}\right] .
\end{gathered}
$$

The following theorem gives the solvability condition for the standard $H^{\infty}$ control problem.

Theorem 1. The standard $H^{\infty}$ control problem is solvable if and only if the following five conditions are satisfied.

(i) $\gamma^{2} I_{r}-D_{11}^{T}\left(I_{m}-D_{12}\left(D_{12}^{T} D_{12}\right)^{-1} D_{12}^{T}\right) D_{11}>0$.

(ii) $\gamma^{2} I_{m}-D_{11}\left(I_{r}-D_{21}^{T}\left(D_{21} D_{21}^{T}\right)^{-1} D_{21}\right) D_{11}^{T}>0$.

(iii) There exists a solution $X \geq 0$ of the Riccati equation
$X A+A^{T} X+C_{z}^{T} C_{z}$

$-\left(C_{z}^{T} D_{z}+X B\right)\left(D_{z}^{T} J_{\gamma} D_{z}\right)^{-1}\left(D_{z}^{T} C_{z}+B^{T} X\right)=0$

such that $A+B F$ is stable, where

$$
F=\left[\begin{array}{c}
F_{w} \\
F_{u}
\end{array}\right]:=-\left(D_{z}^{T} J_{\gamma} D_{z}\right)^{-1}\left(D_{z}^{T} C_{z}+B^{T} X\right) .
$$

(iv) There exists a solution $Y \geq 0$ of the Riccati equation

$$
\begin{aligned}
& Y A^{T}+A Y+B_{w} B_{w}^{T} \\
& -\left(B_{w} D_{w}^{T}+Y C^{T}\right)\left(D_{w} J_{\gamma}^{\prime} D_{w}^{T}\right)^{-1}\left(D_{w} B_{w}^{T}+C Y\right)=0
\end{aligned}
$$

such that $A+L C$ is stable, where

$$
L=\left[\begin{array}{ll}
L_{z} & L_{y}
\end{array}\right]:=-\left(B_{w} D_{w}^{T}+Y C^{T}\right)\left(D_{w} J_{\gamma}^{\prime} D_{w}^{T}\right)^{-1} .
$$

(v) $\lambda_{\max }(X Y)<\gamma^{2}$.

Proof: See THEOREM 8.12 (p. 210) in 11).

\section{Chain-scattering approach to $H^{\infty}$ control}

In this section, we briefly introduce the chain-scattering approach to $H^{\infty}$ control. More details of this approach are found in 11).

If $P_{21}(s)$ is invertible, a necessary and sufficient condition for the solvability is that the chain-scattering form of $P(s)$ has $J$-lossless factorization

$$
\left[\begin{array}{l}
z \\
w
\end{array}\right]=C H A I N(P)\left[\begin{array}{l}
u \\
y
\end{array}\right]=\Theta \Pi\left[\begin{array}{l}
u \\
y
\end{array}\right],
$$

where $\Theta$ is $J$-lossless (see the section 4.4 in 11)) and $\Pi$ is unimodular. Then, the $H^{\infty}$ controller is constructed as

$$
K(s)=H M\left(\Pi^{-1} ; S\right), \quad S \in \boldsymbol{B H}^{\infty} .
$$

Due to the cascade property of $H M(\cdot ; \cdot)$ (see the notation in Section 1), we can obtain the representation of $\Phi(s)$ without using a representation of the closed-loop transfer function $\Pi^{-1}$ explicitly, i.e.,

$$
\Phi(s)=H M(\Theta ; S),
$$

which is described in Fig. 2.

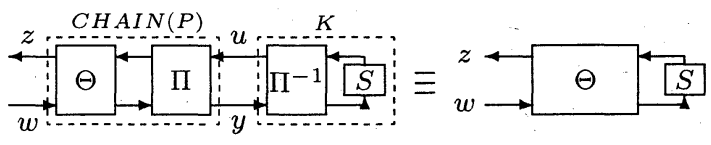

Fig. 2 Structure of $H^{\infty}$ control

If neither $P_{21}(s)$ nor $P_{12}(s)$ is invertible, i.e., in the socalled four-block case, we need to augment the plant to derive chain-scattering representations of $P(s)$. For the plant given by (2), we consider a fictitious output

$$
y^{\prime}=C_{2}^{\prime} x+D_{21}^{\prime} w
$$

where $D_{21}^{\prime}$ is any matrix that makes $\left[\begin{array}{c}D_{21} \\ D_{21}^{\prime}\end{array}\right]$ invertible. The augmented plant $P_{o}$ is described by

$$
\left[\begin{array}{c}
z \\
y \\
y^{\prime}
\end{array}\right]=P_{o}\left[\begin{array}{c}
w \\
u
\end{array}\right]=\left[\begin{array}{c|cc}
A & B_{1} & B_{2} \\
\hline C_{1} & D_{11} & D_{12} \\
\widehat{C}_{2} & \widehat{D}_{21} & 0
\end{array}\right]\left[\begin{array}{l}
w \\
u
\end{array}\right]
$$

where $\widehat{C}_{2}:=\left[\begin{array}{l}C_{2} \\ C_{2}^{\prime}\end{array}\right], \quad \widehat{D}_{21}:=\left[\begin{array}{c}D_{21} \\ D_{21}^{\prime}\end{array}\right]$.

We obtain the chain-scattering representation of $P_{o}$ as 


$$
\begin{aligned}
& {\left[\begin{array}{c}
z \\
w
\end{array}\right]=\operatorname{CHAIN}\left(P_{o}\right)\left[\begin{array}{c}
u \\
y \\
y^{\prime}
\end{array}\right]:=\left[\begin{array}{c|c}
A_{\Sigma} & B_{\Sigma} \\
\hline C_{\Sigma} & D_{\Sigma}
\end{array}\right]\left[\begin{array}{c}
u \\
y \\
y^{\prime}
\end{array}\right]} \\
& =\left[\begin{array}{c|cc}
A-B_{1} \widehat{D}_{21}^{-1} \widehat{C}_{2} & B_{2} & B_{1} \widehat{D}_{21}^{-1} \\
\hline C_{1}-D_{11} \widehat{D}_{21}^{-1} \widehat{C}_{2} & D_{12} & D_{11} \widehat{D}_{21}^{-1} \\
-\widehat{D}_{21}^{-1} \widehat{C}_{2} & 0 & \widehat{D}_{21}^{-1}
\end{array}\right]\left[\begin{array}{c}
u \\
y \\
y^{\prime}
\end{array}\right] .
\end{aligned}
$$

Hereafter, the matrices which include the augmentation $\left(C_{2}^{\prime}, D_{21}^{\prime}\right)$ are designated by a symbol " $\wedge$ ".

Now, we introduce partial plants which are given by $P_{z}:=\left[\begin{array}{cc}P_{11} & P_{12} \\ I_{r} & 0\end{array}\right]=\left[\begin{array}{c|c}A & B \\ \hline C_{z} & D_{z}\end{array}\right], \widehat{P}_{y}:=\left[\begin{array}{cc}0 & I_{p} \\ \widehat{P}_{21} & \widehat{P}_{22}\end{array}\right]=\left[\begin{array}{c|c}A & B \\ \widehat{C}_{y} & \widehat{D}_{y}\end{array}\right]$,

where $\quad \widehat{C}_{y}:=\left[\begin{array}{c}0 \\ \widehat{C}_{2}\end{array}\right], \quad \widehat{D}_{y}:=\left[\begin{array}{cc}0 & I_{p} \\ \widehat{D}_{21} & 0\end{array}\right]$.

Using these partial plants, we can obtain an alternative representation of $C H A I N\left(P_{o}\right)$ in (14) as

$$
\left[\begin{array}{c|c}
A_{\Sigma} & B_{\Sigma} \\
\hline C_{\Sigma} & D_{\Sigma}
\end{array}\right]=\left[\begin{array}{c|c}
A-B \widehat{D}_{y}^{-1} \widehat{C}_{y} & B \widehat{D}_{y}^{-1} \\
\hline C_{z}-D_{z} \widehat{D}_{y}^{-1} \widehat{C}_{y} & D_{z} \widehat{D}_{y}^{-1}
\end{array}\right] .
$$

Next, we consider the $J$-lossless factorization (9) for the augmented plant (16). For this purpose the following theorem is now available ${ }^{11)}$.

Theorem 2. Let (16) be a minimal realization of $C H A I N\left(P_{o}\right)$ with $D_{\Sigma} \in \Re^{(m+r) \times(p+r)}$. It has a $\left(J_{m r}, J_{p r}\right)$-lossless factorization if and only if the following three conditions are satisfied.

(i) There exists a nonsingular matrix $E_{\Sigma}$ satisfying

$$
D_{\Sigma}^{T} J_{\gamma} D_{\Sigma}=E_{\Sigma}^{T} J_{p r} E_{\Sigma}
$$

(ii) There exists a solution $X \geq 0$ of the Riccati equation

$$
\begin{aligned}
& X A_{\Sigma}+A_{\Sigma}^{T} X-\left(C_{\Sigma}^{T} J_{\gamma} D_{\Sigma}+X B_{\Sigma}\right)\left(D_{\Sigma}^{T} J_{\gamma} D_{\Sigma}\right)^{-1} \\
& \cdot\left(D_{\Sigma}^{T} J_{\gamma} C_{\Sigma}+B_{\Sigma}^{T} X\right)+C_{\Sigma}^{T} C_{\Sigma}=0
\end{aligned}
$$

such that $\widehat{A}_{\Sigma}=A_{\Sigma}+B_{\Sigma} F_{\Sigma}$ is stable, where

$$
F_{\Sigma}=-\left(D_{\Sigma}^{T} J_{\gamma} D_{\Sigma}\right)^{-1}\left(D_{\Sigma}^{T} J_{\gamma} C_{\Sigma}+B_{\Sigma}^{T} X\right) \text {. }
$$

(iii) There exists a solution $\bar{X} \geq 0$ of the Riccati equation

$$
\bar{X} A_{\Sigma}^{T}+A_{\Sigma} \bar{X}+\bar{X} C_{\Sigma}^{T} J_{\gamma} C_{\Sigma} \bar{X}=0
$$

such that $\bar{A}_{\Sigma}=A_{\Sigma}+\bar{X} C_{\Sigma}^{T} J_{\gamma} C_{\Sigma}$ is stable, and

$$
\lambda_{\max }(X \bar{X})<1 \text {. }
$$

In this case, the $\left(J_{m r}, J_{p r}\right)$-lossless factor is given by

$$
\begin{aligned}
& \Theta=\left[\begin{array}{ll}
\Theta_{11} & \Theta_{12} \\
\Theta_{21} & \Theta_{22}
\end{array}\right]= \\
& {\left[\begin{array}{c|c}
-\bar{A}_{\Sigma}^{T} & 0 \\
0 & A_{\Sigma}+B_{\Sigma} F_{\Sigma}
\end{array} \mid\left[\begin{array}{cc}
I & -X \\
-\bar{X} & I
\end{array}\right]^{-1}\left[\begin{array}{c}
C_{\Sigma}^{T} J_{\gamma} D_{\Sigma} \\
B_{\Sigma}
\end{array}\right] E_{\Sigma}^{-1} .\right.}
\end{aligned}
$$

Proof: See THEOREM 6.6 (p. 139) in Kimura ${ }^{11)}$.

In this paper, we deal with the closed-loop transfer function resulting from the central solution. This is equivalent to putting $S=0$ in (11), which implies

$$
\Phi(s)=H M\left(\left[\begin{array}{ll}
\Theta_{11} & \Theta_{12} \\
\Theta_{21} & \Theta_{22}
\end{array}\right] ; 0\right)=\Theta_{12} \Theta_{22}^{-1},
$$

where $\Theta_{12}$ and $\Theta_{22}$ are given by (21).

Now, we introduce the notion of maximum augmentation ${ }^{11)}$. Though Theorem 2 is not a sufficient condition for the solvability of $H^{\infty}$ control problem for the original plant, it is shown that a sufficient condition is derived for a special augmentation which we call maximum augmentation.

Definition 1. The output augmentation $\left(C_{2}^{\prime}, D_{21}^{\prime}\right)$ in (12) is said to be maximum augmentation, if $\left(C_{2}^{\prime}, D_{21}^{\prime}\right)$ satisfies

$$
D_{21}^{\prime}\left(B_{1}+L\left[\begin{array}{c}
D_{11} \\
D_{21}
\end{array}\right]\right)^{T}+C_{2}^{\prime} Y=0
$$

where $Y$ is the solution of the Riccati equation (7).

Similarly, if $P_{12}(s)$ is not invertible, we can consider a dual notion of an output augmentation in (12) as $\left(B_{2}^{\prime}, D_{12}^{\prime}\right)$. We define an input augmented plant as

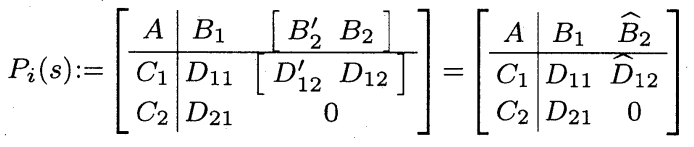

The maximum input augmentation which is a complete dualization of (23) is also defined as follows.

Definition 2. The input augmentation $\left(B_{2}^{\prime}, D_{12}^{\prime}\right)$ in (24) is said to be maximum augmentation, if $\left(B_{2}^{\prime}, D_{12}^{\prime}\right)$ satisfies

$$
\left(C_{1}+\left[\begin{array}{ll}
D_{11} & D_{12}
\end{array}\right] F\right)^{T} D_{12}^{\prime}+X B_{2}^{\prime}=0
$$

where $X$ is the solution of the Riccati equation (6). We shall also give a new interpretation of the maximum augmentation in the next section.

\section{Main results}

In this section we show that the McMillan degree of the closed-loop transfer function is determined by the sum of the number of stable invariant zeros of $P_{12}(s)$ and $P_{21}(s)$, which are parts of the original plant.

\section{1 Closed-loop structure of the central $H^{\infty}$ controller}

In order to prove the main theorem, we show a few lemmas which give several properties of maximum augmentation of the plant. Note that, though we only deal with the output augmentation $\left(C_{2}^{\prime}, D_{21}^{\prime}\right)$ in this section, the complete dualization (input augmentation) of our results also holds.

We consider a plant $P(s)$ in (2) for which $H^{\infty}$ control problem is solvable. Assume that both $P_{12}(s)$ and $P_{21}(s)$ are not invertible, i.e., consider the four-block case. Then, provided the maximum augmentation in (23) is chosen for $\left(C_{2}^{\prime}, D_{21}^{\prime}\right)$, the $H^{\infty}$ control problem on the augmented plant $P_{o}(s)$ in (13) is also solvable. Hence, the solvability conditions (i)-(v) in Theorem 2 are satisfied. Substituting (16) into (20) and $\bar{A}_{\Sigma}$ of the condition (iii), we have

$$
\begin{array}{r}
\left(A-B_{1} \widehat{D}_{21}^{-1} \widehat{C}_{2}\right) Y+Y\left(A-B_{1} \widehat{D}_{21}^{-1} \widehat{C}_{2}\right)^{T} \\
-Y \widehat{C}^{T}\left(\widehat{D}_{w} J_{\gamma}^{\prime} \widehat{D}_{w}^{T}\right)^{-1} \widehat{C} Y=0, \\
\bar{A}_{\Sigma}=A-B_{1} \widehat{D}_{21}^{-1} \widehat{C}_{2}-Y \widehat{C}^{T}\left(\widehat{D}_{w} J_{\gamma}^{\prime} \widehat{D}_{w}^{T}\right)^{-1} \widehat{C},
\end{array}
$$

where $\widehat{C}:=\left[\begin{array}{l}C_{1} \\ \widehat{C}_{2}\end{array}\right], \quad \widehat{D}_{w}:=\left[\begin{array}{ll}D_{11} & I \\ \widehat{D}_{21} & 0\end{array}\right]$. Here, in order to derive (26) and (27), we used the identities 
$A_{\Sigma}=A-B_{1} \widehat{D}_{21}^{-1} \widehat{C}_{2}, C_{\Sigma}=\left[\begin{array}{cc}0 & I_{m} \\ -I_{r} & 0\end{array}\right] \widehat{D}_{w}^{-1} \widehat{C}, Y=\gamma^{2} \bar{X} .(28)$

As regards (26) and (27), we can prove the following lemmas.

Lemma 1. If $\left(C_{2}^{\prime}, D_{21}^{\prime}\right)$ in $P_{o}(s)$ is the maximum augmentation given by (23), the eigenvalues of $A-B_{1} \widehat{D}_{21}^{-1} \widehat{C}_{2}$ are independent of the augmentation $\left(C_{2}^{\prime}, D_{21}^{\prime}\right)$.

Proof: The Hamiltonian matrix corresponding to the Riccati equation (26) is given by

$$
H:=\left[\begin{array}{cc}
\left(A-B_{1} \widehat{D}_{21}^{-1} \widehat{C}_{2}\right)^{T} & -\widehat{C}^{T}\left(\widehat{D}_{w} J_{\gamma}^{\prime} \widehat{D}_{w}^{T}\right)^{-1} \widehat{C} \\
0 & -\left(A-B_{1} \widehat{D}_{21}^{-1} \widehat{C}_{2}\right)
\end{array}\right] .
$$

Obviously, we have

$\lambda\{H\}=\lambda\left\{A-B_{1} \widehat{D}_{21}^{-1} \widehat{C}_{2}\right\} \cup \lambda\left\{-\left(A-B_{1} \widehat{D}_{21}^{-1} \widehat{C}_{2}\right)\right\}$,

where $\lambda\{\cdot\}$ denotes a set of eigenvalues.

On the other hand, from the definition of $L$, we have

$$
L D_{w} J_{\gamma}^{\prime} D_{w}^{T}=-B_{w} D_{w}^{T}-Y C^{T} .
$$

From the maximum augmentation (23), it follows that $L D_{w} J_{\gamma}^{\prime}\left[\begin{array}{ll}D_{21}^{\prime} & 0\end{array}\right]^{T}=-B_{w}\left[D_{21}^{\prime} 0\right]^{T}-Y C_{2}^{\prime T}$.

From (31) and (32), we obtain

$$
B_{w} \widehat{D}_{w}^{T}+Y \widehat{C}^{T}=-L D_{w} J_{\gamma}^{\prime} \widehat{D}_{w}^{T} .
$$

Noting that $B_{1} \widehat{D}_{21}^{-1} \widehat{C}_{2}=B_{w} \widehat{D}_{w}^{T}\left(\widehat{D}_{w} J_{\gamma}^{\prime} \widehat{D}_{w}^{T}\right)^{-1} \widehat{C}$ and (33), we calculate $\bar{A}_{\Sigma}$ in (27) as

$$
\begin{aligned}
\bar{A}_{\Sigma} & =A-\left(B_{w} \widehat{D}_{w}^{T}+Y \widehat{C}^{T}\right)\left(D_{w} J_{\gamma}^{\prime} \widehat{D}_{w}^{T}\right)^{-1} \widehat{C} \\
& =A+L D_{w} \widehat{D}_{w}^{-1} \widehat{C}=A+L C .
\end{aligned}
$$

From (26) and (34), the Hamiltonian in (29) is given by $H=$

$$
\begin{aligned}
& {\left[\begin{array}{cc}
I & 0 \\
Y & I
\end{array}\right]\left[\begin{array}{cc}
\left(A-B_{1} \widehat{D}_{21}^{-1} \widehat{C}_{2}\right)^{T} & -\widehat{C}^{T}\left(\widehat{D}_{w} J_{\gamma}^{\prime} \widehat{D}_{w}^{T}\right)^{-1} \widehat{C} \\
0 & -\left(A-B_{1} \widehat{D}_{21}^{-1} \widehat{C}_{2}\right)
\end{array}\right]\left[\begin{array}{cc}
I & 0 \\
-Y & I
\end{array}\right]} \\
& =\left[\begin{array}{cc}
I & 0 \\
Y & I
\end{array}\right]\left[\begin{array}{cc}
\bar{A}_{\Sigma}^{T} & * \\
0 & -\bar{A}_{\Sigma}
\end{array}\right]\left[\begin{array}{cc}
I & 0 \\
-Y & I
\end{array}\right] \\
& =\left[\begin{array}{cc}
I & 0 \\
Y & I
\end{array}\right]\left[\begin{array}{cc}
(A+L C)^{T} & * \\
0 & -(A+L C)
\end{array}\right]\left[\begin{array}{cc}
I & 0 \\
-Y & I
\end{array}\right]
\end{aligned}
$$

From (30) and (35), we have

$$
\lambda\{H\}=\lambda\{A+L C\} \cup \lambda\{-(A+L C)\}
$$

which is independent of $\left(C_{2}^{\prime}, D_{21}^{\prime}\right)$.

Lemma 2. The Riccati equation (26) has a stabilizing solution $Y \geq 0$ only if $A-B_{1} \widehat{D}_{21}^{-1} \widehat{C}_{2}$ has no eigenvalue on the imaginary axis. The rank of $Y$ is equal to the number of unstable eigenvalues of $A-B_{1} \widehat{D}_{21}^{-1} \widehat{C}_{2}$ and the eigenspace of $A-B_{1} \widehat{D}_{21}^{-1} \widehat{C}_{2}$ corresponding to the stable eigenvalues is equal to $\operatorname{Ker} Y$.

Proof: Apply LEMMA 3.6 (p. 49) ${ }^{11)}$ to (26).

Lemma 2 implies that the rank of the stabilizing solution to the Riccati equation (7) coincides with the number of unstable eigenvalues of $A-B_{1} \widehat{D}_{21}^{-1} \widehat{C}_{2}$. The following lemma relates the zeros of the original plant $P_{21}$ to those of the augmented plant $\widehat{P}_{21}$, i.e., the eigenvalues of $A-B_{1} \widehat{D}_{21}^{-1} \widehat{C}_{2}$.

Lemma 3. If $\widehat{P}_{21}$ is obtained by the maximum output augmentation, then all zeros of $\widehat{P}_{21}$ except for the zeros of $P_{21}$ are unstable.
Proof: We prove that if a zero of $\widehat{P}_{21}$ is stable, the zero is one of $P_{21}$. Let

$$
P_{w}:=\left[\begin{array}{ll}
P_{11} & I \\
P_{21} & 0
\end{array}\right]=\left[\begin{array}{c|c}
A & B_{w} \\
\hline C & D_{w}
\end{array}\right], \quad \widehat{P}_{w}:=\left[\begin{array}{cc}
P_{11} & I \\
\widehat{P}_{21} & 0
\end{array}\right]=\left[\begin{array}{c|c}
A & B_{w} \\
\hline \widehat{C} & \widehat{D}_{w}
\end{array}\right] .
$$

By the definition of invariant zero, the zeros of $P_{21}$ and $\widehat{P}_{21}$ coincide with those of $P_{w}$ and $\widehat{P}_{w}$, respectively. Hence, if $\lambda(\operatorname{Re} \lambda<0)$ is a stable zero of $\widehat{P}_{21}$ then $\lambda$ is also a zero of $\widehat{P}_{w}$. Since $\widehat{P}_{w}$ is invertible, $\lambda$ is calculated as the eigenvalue of $A-B_{1} \widehat{D}_{21}^{-1} \widehat{C}_{2}$, i.e., there exists $\eta \neq 0$ such that

$$
\left(A-B_{1} \widehat{D}_{21}^{-1} \widehat{C}_{2}\right)^{T} \eta=\lambda \eta .
$$

Lemma 2 implies $\eta \in \operatorname{Ker} Y$. Therefore, by multiplying $\eta^{T}$ to (34), we have

$$
\eta^{T}\left\{A-B_{w} D_{w}^{T}\left(D_{w} J_{\gamma}^{\prime} D_{w}^{T}\right)^{-1} C\right\}=\lambda \eta^{T} .
$$

On the other hand, multiplying $\eta^{T}$ and $\left(J_{\gamma}^{\prime} \widehat{D}_{w}^{T}\right)^{-1}$ to (33) yields

$$
\eta^{T} B_{w} D_{w}^{T}\left(D_{w} J_{\gamma}^{\prime} D_{w}^{T}\right)^{-1} D_{w}=\eta^{T} B_{w},
$$

where, we used the definition of $L$ given by (8) and the relation $B_{w} J_{\gamma}^{\prime-1}=B_{w}$. From (39) and (40), we obtain

$$
\left[\begin{array}{ll}
\eta^{T} & \eta^{T} B_{w} D_{w}^{T}\left(D_{w} J_{\gamma}^{\prime} D_{w}^{T}\right)^{-1}
\end{array}\right]\left[\begin{array}{cc}
\lambda I-A & -B_{w} \\
C & D_{w}
\end{array}\right]=0,
$$

which implies that $\lambda$ is the invariant zero of $P_{w}(s)$.

Remark: In the $H^{\infty}$ control problem, the partial plant $P_{21}\left(P_{12}\right)$ is generally fat (tall) and is not invertible. Hence, $P_{21}\left(P_{12}\right)$ may not have $n$ invariant zeros, whereas the augmented plant $\widehat{P}_{21}\left(\widehat{P}_{12}{ }^{(2)}\right)$ has $n$ invariant zeros. The above lemma implies that the maximum augmentation does not add new stable zeros to the zeros of $\widehat{P}_{21}$ and $\widehat{P}_{12}$. Hence, due to Lemma 2, the rank of the stabilizing solution $Y(X)$ is equal to $n-\#\left\{\right.$ the stable zeros of $\left.P_{21}\left(P_{12}\right)\right\}$.

Now, we are ready to show one of our main results, which is on the degree of the closed-loop system.

Theorem 3. In addition to the assumptions (A1) and (A2), assume that an $H^{\infty}$ control problem for a plant $P(s)$ is solvable. Let the number of the stable invariant zeros of $P_{12}(s)$ and $P_{21}(s)$ be $\rho_{12}$ and $\rho_{21}$, respectively. Then, the McMillan degree of the closed-loop transfer function is at most $2 n-\left(\rho_{12}+\rho_{21}\right)$.

Proof: Due to the assumption (A2), we can always find nonsingular matrices $T_{Y}$ and $T_{X}$ such that

$$
\begin{aligned}
T_{Y}^{T}\left(A-B_{1} \widehat{D}_{21}^{-1} \widehat{C}_{2}\right) T_{Y}^{-T} & =\left[\begin{array}{cc}
\Lambda_{Y+} & 0 \\
0 & \Lambda_{Y-}
\end{array}\right], \\
T_{X}^{-1}\left(A-\widehat{B}_{2} \widehat{D}_{12}^{-1} C_{1}\right) T_{X} & =\left[\begin{array}{cc}
\Lambda_{X+} & 0 \\
0 & \Lambda_{X-}
\end{array}\right],
\end{aligned}
$$

where both $-\Lambda_{+_{+}}$and $\Lambda_{-_{-}}$are stable. From Lemma 1, $-\Lambda_{+}$and $\Lambda_{-}$are independent of the augmentations $\left(C_{2}^{\prime}, D_{21}^{\prime}\right)$ and $\left(B_{2}^{\prime}, D_{12}^{\prime}\right)$, respectively. From Lemma 2,

(2) $\widehat{P}_{12}$ includes the maximum input augmentation in (25). 
the size of $\Lambda_{X+}$ is $n-\rho_{12}$ and the size of $\Lambda_{Y+}$ is $n-\rho_{21}$.

Divide $T_{Y}$ as

$$
T_{Y}=:\left[\begin{array}{ll}
T_{Y 1} & T_{Y 2}
\end{array}\right] \text {, }
$$

where the number of columns of $T_{Y 1}$ is $n-\rho_{21}$. From (42), it follows that

$$
\left(A-B_{1} \widehat{D}_{21}^{-1} \widehat{C}_{2}\right)^{T} T_{Y 2}=T_{Y 2} \Lambda_{Y-}^{T} .
$$

Due to Lemma 2, we obtain $Y T_{Y 2}=0$. Therefore, we have

$$
T_{Y}^{T} Y T_{Y}=\left[\begin{array}{c}
T_{Y 1}^{T} \\
T_{Y 2}^{T}
\end{array}\right] Y\left[\begin{array}{ll}
T_{Y 1} & T_{Y 2}
\end{array}\right]=\left[\begin{array}{cc}
T_{Y 1}^{T} Y T_{Y 1} & 0 \\
0 & 0
\end{array}\right]
$$

Furthermore, we can show that $Y_{0}:=T_{Y 1}^{T} Y T_{Y 1}$ is positive definite. Dualization of (46) yields

$$
T_{X}^{T} X T_{X}=\left[\begin{array}{cc}
X_{0} & 0 \\
0 & 0
\end{array}\right], \quad X_{0}: \text { nonsingular },
$$

where the size of $X_{0}$ is $n-\rho_{12}$.

For the $J$-lossless system $\Theta(s)$ given by (21), we, will consider its similarity transformation using

$$
T:=\left[\begin{array}{cc}
T_{Y} & 0 \\
0 & T_{X}
\end{array}\right] \text {. }
$$

in the sequel. Using (27), (42) and (46), we have $T_{Y}^{T} \bar{A}_{\Sigma} T_{Y}^{-T}=T_{Y}^{T}\left(A-B_{1} \widehat{D}_{21}^{-1} \widehat{C}_{2}\right) T_{Y}^{-T}$

$$
\begin{gathered}
-T_{Y}^{T} Y T_{Y} \cdot T_{Y}^{-1} \widehat{C}^{T}\left(\widehat{D}_{w}^{\prime} J_{\gamma}^{\prime} \widehat{D}_{w}^{T}\right)^{-1} \widehat{C} T_{Y}^{-T} \\
=\left[\begin{array}{cc}
\Lambda_{Y+} & 0 \\
0 & \Lambda_{Y-}
\end{array}\right]-\left[\begin{array}{cc}
Y_{0} & 0 \\
0 & 0
\end{array}\right] T_{Y}^{-1} \widehat{C}^{T}\left(\widehat{D}_{w} J_{\gamma}^{\prime} \widehat{D}_{w}^{T}\right)^{-1} \widehat{C} T_{Y}^{-T} \\
=\left[\begin{array}{cc}
\Lambda_{Y+}-Y_{0} \bar{T}_{Y 1} \widehat{C}^{T}\left(\widehat{D}_{w} J_{\gamma}^{\prime} \widehat{D}_{w}^{T}\right)^{-1} \widehat{C} T_{Y}^{-T} & * \\
0 & \Lambda_{Y-}
\end{array}\right],
\end{gathered}
$$

where $\bar{T}_{Y 1}:=\left[\begin{array}{ll}I_{n-\rho_{21}} & 0\end{array}\right] T_{Y}^{-1}$. Dualization of (49) yields $T_{X}^{-1}\left(A_{\Sigma}+B_{\Sigma} F_{\Sigma}\right) T_{X}$

$=\left[\begin{array}{cc}\Lambda_{X+} & 0 \\ 0 & \Lambda_{X-}\end{array}\right]-T_{X}^{-1} \widehat{B}\left(\widehat{D}_{z}^{T} J_{\gamma} \widehat{D}_{z}\right)^{-1} \widehat{B}^{T} T_{X}^{T}\left[\begin{array}{cc}X_{0} & 0 \\ 0 & 0\end{array}\right]$

$=\left[\begin{array}{cc}\Lambda_{X+}-\bar{T}_{X 1} \widehat{B}\left(\widehat{D}_{z}^{T} J_{\gamma} \widehat{D}_{z}\right)^{-1} \widehat{B}^{T} T_{X 1}^{T} X_{0} & 0 \\ * & \Lambda_{Y-}\end{array}\right]$,

where $\bar{T}_{X 1}:=\left[I_{n-\rho_{12}} 0\right] T_{X}^{-1}$. Therefore, the $A$-matrix of $\Theta$ in (21) is transformed as

$$
\begin{aligned}
& T^{-1}\left[\begin{array}{cc}
-\bar{A}_{\Sigma}^{T} & 0 \\
0 & A_{\Sigma}+B_{\Sigma} F_{\Sigma}
\end{array}\right] T \\
& =\left[\begin{array}{cc}
-T_{Y}^{-1} \bar{A}_{\Sigma}^{T} T_{Y} & 0 \\
0 & T_{X}^{-1}\left(A_{\Sigma}+B_{\Sigma} F_{\Sigma}\right) T_{X}
\end{array}\right] \\
& =\left[\begin{array}{cc}
{\left[\begin{array}{cc}
* & 0 \\
* & -\Lambda_{Y-}^{T}
\end{array}\right]} & 0 \\
0 & {\left[\begin{array}{cc}
* & 0 \\
* & \Lambda_{X-}
\end{array}\right]}
\end{array}\right]
\end{aligned}
$$

From dualization of (40), we obtain the identity

$$
\left\{C_{z}-D_{z}\left(D_{z}^{T} J_{\gamma} D_{z}\right)^{-1} D_{z}^{T} C_{z}\right\} T_{X 2}=0,
$$

where $T_{X 2}:=T_{X}\left[\begin{array}{ll}0 & I_{\rho_{12}}\end{array}\right]^{T}$ consists of the eigenvectors of $A-\widehat{B}_{2} \widehat{D}_{12}^{-1} C_{1}$ corresponding to the stable part $\Lambda_{X-}$ in (43).

On the other hand, routine calculations using (16) and (19) yield

$$
F_{\Sigma}=\widehat{C}_{y}+\widehat{D}_{y} F
$$

Using this relation and (52), we have

$$
\begin{aligned}
& \left(C_{\Sigma}+D_{\Sigma} F_{\Sigma}\right) T_{X} \\
& =\left\{C_{z}-D_{z} \widehat{D}_{y}^{-1} \widehat{C}_{y}+D_{z} \widehat{D}_{y}^{-1}\left(\widehat{C}_{y}+\widehat{D}_{y} F\right)\right\} T_{X} \\
& =\left(C_{z}+D_{z} F\right) T_{X} \\
& =\left[\left\{C_{z}-D_{z}\left(D_{z}^{T} J_{\gamma} D_{z}\right)^{-1} D_{z}^{T} C_{z}\right\} T_{X 1} 0\right] \\
& -D_{z}\left(D_{z}^{T} J_{\gamma} D_{z}\right)^{-1} B^{T} T_{X}^{-T}\left[\begin{array}{cc}
X_{0} & 0 \\
0 & 0
\end{array}\right],
\end{aligned}
$$

where $T_{X 1}:=T_{X}\left[I_{n-\rho_{12}} 0\right]^{T}$. Thẹrefore, from (46) and (54), the $C$-matrix of $\Theta$ is transformed as

$$
\begin{aligned}
& {\left[\begin{array}{l}
-\gamma^{-2} C_{\Sigma} Y T_{Y} \\
\left(C_{\Sigma}+D_{\Sigma} F_{\Sigma}\right) T_{X}
\end{array}\right]} \\
& =\left[-\gamma^{-2} C_{\Sigma} T_{Y}^{-T}\left[\begin{array}{cc}
Y_{0} & 0 \\
0 & 0
\end{array}\right]\left[\begin{array}{ll}
* & 0 \\
* & 0
\end{array}\right]\right] .
\end{aligned}
$$

Hence, all modes corresponding to $-\Lambda_{Y-}^{T}$ and $\Lambda_{X-}$ in (51) are unobservable. As a result, we obtain the $J$ lossless system $\Theta(s)$ whose McMillan degree is at most $2 n-\left(\rho_{12}+\rho_{21}\right)$. It can be shown that the closed-loop system with the central $H^{\infty}$ controller does not have a greater McMillan degree than the $J$-lossless system $\Theta(s)$. The proof is completed.

For the one-block case, we can obtain the following result.

Corollary 1. Under the assumptions (A1) and (A2), assume that an $H^{\infty}$ control problem in the one-block case is solvable. Let the number of unstable invariant zeros of $P_{12}(s)$ and $P_{21}(s)$ be $\rho_{12}^{+}$and $\rho_{21}^{+}$, respectively. Then, the McMillan degree of the closed-loop transfer function given by $(61)$ is at most $\rho_{12}^{+}+\rho_{21}^{+}$.

As a direct consequence of the Theorem 4, the case where $\rho_{12}=\rho_{21}=n$ is of particular interest.

Corollary 2. Under the assumptions (A1) and (A2), assume that the $H^{\infty}$ control problem is solvable. If both $P_{12}(s)$ and $P_{21}(s)$ have $n$ stable invariant zeros, i. e., $\rho_{12}=\rho_{21}=n$, then the closed-loop transfer function is given by

$$
\Phi(s)=\left\{I_{m}-D_{12}\left(D_{12}^{T} D_{12}\right)^{-1} D_{12}^{T}\right\} D_{11},
$$

which has no dynamics. In particular, the closed-loop transfer function satisfies $\Phi(s)=0$ in the one-block case, which shows that the $H^{\infty}$ control problem is solvable for any $\gamma>0$.

Proof: Since both $\widehat{P}_{12}(s)$ and $\widehat{P}_{21}(s)$ have no unstable zeros, we have $X=Y=0$ and

$$
\begin{aligned}
T_{X}^{-1}\left(A-\widehat{B}_{2} \widehat{D}_{12}^{-1} C_{1}\right) T_{X} & =\Lambda_{X-}, \\
T_{Y}^{T}\left(A-B_{1} \widehat{D}_{21}^{-1} \widehat{C}_{2}\right) T_{Y}^{-T} & =\Lambda_{Y-} .
\end{aligned}
$$

Using the similarity transformation $T$ in (48), the $J$ lossless system $\Theta(s)$ in (81) is given by

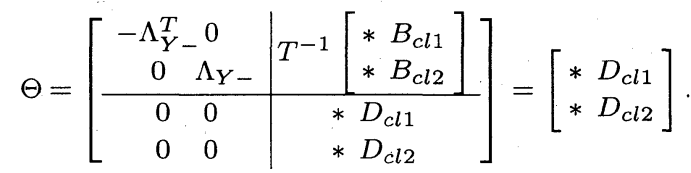


Hence, we obtain the closed-loop transfer function as

$$
\begin{aligned}
\Phi(s) & =\Theta_{12} \Theta_{22}^{-1}=D_{c l 1} D_{c l 2}^{-1} \\
& =\left\{I_{m}-D_{12}\left(D_{12}^{T} D_{12}\right)^{-1} D_{12}^{T}\right\} D_{11} .
\end{aligned}
$$

Remark: Theorem 3 demonstrates an interesting link between $H^{\infty}$ control and $J$-lossless conjugation ${ }^{11)}$. Theorem 3 shows that, in $H^{\infty}$ control, the order of closed-loop transfer function depends on the number of the stable invariant zeros of $P_{21}(s)$ and $P_{12}(s)$. It may not be equal to the order of the plant. In fact, $2 n-\left(\rho_{12}+\rho_{21}\right)$ equals to the sum of the numbers of pole extractions and zero extractions for $C H A I N(P(s))$, which are key procedures of $J$-lossless factorization based on $J$-lossless conjugation. This is a significant characteristic feature of $H^{\infty}$ control.

Remark: The result of Theorem 3 holds in the generic case (A property on the original plant, ( $A, B_{1}, B_{2}, C_{1}, C_{2}, D_{11}, D_{12}, D_{21}$ ), is said to be generic if the property holds for almost all plants except special ones.). In fact, we can find an example whose degree of the closed-loop system is exactly $2 n-\left(\rho_{12}+\rho_{12}\right)$, which implies that $2 n-\left(\rho_{12}+\rho_{12}\right)$ is lower bound in the generic sense.

4. 2 Representation of the closed-loop transfer function with the central $H^{\infty}$ controller

Now, we are ready to give an explicit state-space representation for the closed-loop transfer function $\Phi(s)$ in (22). Note that this representation does not include the augmentation whereas the $J$-lossless system given by (21) depends on the augmentation.

Theorem 4. Under the assumptions (A1) and (A2), if $H^{\infty}$ control problem is solvable, the closed-loop transfer function for the central controller in (22) is described by

$$
\begin{aligned}
\Phi(s) & =\Theta_{12} \Theta_{22}^{-1}=\left[\begin{array}{l|l}
A_{c l} & B_{c l} \\
\hline C_{c l} & D_{c l}
\end{array}\right], \\
A_{c l} & =\left[\begin{array}{cc}
-\left\{\begin{array}{c}
T_{Y 1}^{T}(A+L C) \bar{T}_{Y 1}^{T} \\
0
\end{array}\right. & 0 \\
0 & \bar{T}_{X 1}(A+B F) T_{X 1}
\end{array}\right] \\
& +B_{c l}\left[\begin{array}{ll}
-\gamma^{-2}\left(B_{1}+L_{z} D_{11}+L_{y} D_{21}\right)^{T} T_{Y 1} & F_{w} T_{X 1}
\end{array}\right], \\
B_{c l} & =\left[\begin{array}{l}
B_{c l 1} \\
B_{c l 2}
\end{array}\right], \quad C_{c l}=\left[\begin{array}{ll}
C_{c l 1} & C_{c l 2}
\end{array}\right], \\
D_{c l} & =\left(I_{m}-D_{12} D_{12}^{\dagger}\right) D_{11},
\end{aligned}
$$

where $T_{Y 1}:=T_{Y}\left[\begin{array}{c}I_{n-\rho_{21}} \\ 0\end{array}\right], T_{X 1}:=T_{X}\left[\begin{array}{c}I_{n-\rho_{12}} \\ 0\end{array}\right]$,

$\bar{T}_{Y 1}:=\left[I_{n-\rho_{21}} 0\right] T_{Y}^{-1}, \bar{T}_{X 1}:=\left[I_{n-\rho_{12}} 0\right] T_{X}^{-1}$, $D_{12}^{\dagger}:=\left(D_{12}^{T} D_{12}\right)^{-1} D_{12}^{T}, \quad W:=\left(I-\gamma^{-2} Y X\right)^{-1}$,

$$
\begin{aligned}
& B_{c l 1}=\bar{T}_{Y 1}\left(I-\gamma^{-2} X Y\right)^{-1}\left\{X\left(B_{1}-B_{2} D_{12}^{\dagger} D_{11}\right)\right. \\
& \left.-\gamma^{2} T_{Y 1} Y_{0}^{-1} T_{Y 1}^{T}\left(B_{1}+L_{y} D_{21}+L_{z} D_{12} D_{12}^{\dagger} D_{11}\right)\right\}, \\
& B_{c l 2}=-\bar{T}_{X 1} W\left\{\left(B_{2}+L_{z} D_{12}\right) D_{12}^{\dagger} D_{11}+L_{y} D_{21}\right\}, \\
& C_{c l 1}=\left\{-L_{z}^{T}+\gamma^{-2}\left(I_{m}-D_{12} D_{12}^{\dagger}\right) D_{11} .\right.
\end{aligned}
$$

$$
\begin{array}{r}
\left.\left(B_{1}+L_{z} D_{11}+L_{y} D_{21}\right)^{T}\right\} T_{Y 1}, \\
C_{c l 2}=\left(C_{1}+D_{12} F_{u}+D_{12} D_{12}^{\dagger} D_{11} F_{w}\right) T_{X 1} .
\end{array}
$$

Proof: The proof consists of the following three steps. First, we transform the $J$-lossless system $\Theta$ in (21) by using the similarity transformation $T$ given by (48). We show that the $J$-lossless system $\Theta$ whose order is $2 n-$ $\left(\rho_{12}+\rho_{21}\right)$ does not include any augmentations. Finally, using this representation of $\Theta$, we derive the closed-loop system $\Phi$ from (22).

[Step 1] A-matrix of $\Theta$ : From (16) and (53), it follows that

$$
\begin{aligned}
A_{\Sigma}+B_{\Sigma} F_{\Sigma} & =A-B \widehat{D}_{y}^{-1} \widehat{C}_{y}+B \widehat{D}_{y}^{-1}\left(\widehat{C}_{y}+\widehat{D}_{y} F\right) \\
& =A+B F .
\end{aligned}
$$

From (34) and (62), the $A$-matrix of $\Theta$ in (21) is given by

$$
\begin{aligned}
& T^{-1}\left[\begin{array}{cc}
-\bar{A}_{\Sigma}^{T} & 0 \\
0 & A_{\Sigma}+B_{\Sigma} F_{\Sigma}
\end{array}\right] T \\
& =\left[\begin{array}{cc}
-\left\{T_{Y}^{T}(A+L C) T_{Y}^{-T}\right\}^{T} & 0 \\
0 & T_{X}^{-1}(A+B F) T_{X}
\end{array}\right],
\end{aligned}
$$

where the similarity transformation $T$ is given by (48). Based on Theorem 3, we eliminate the unobservable part of $(63)$ to have

$\left[\begin{array}{cc}-\bar{T}_{Y 1}(A+L C)^{T} T_{Y 1} & 0_{\left(n-\rho_{21}\right) \times\left(n-\rho_{12}\right)} \\ 0_{\left(n-\rho_{12}\right) \times\left(n-\rho_{21}\right)} & \bar{T}_{X 1}(A+B F) T_{X 1}\end{array}\right]=:\left[\begin{array}{cc}A_{\theta 1} & 0 \\ 0 & A_{\theta 2}\end{array}\right]$,

where the definition of $T_{1}$ and $\bar{T}_{1}$ is given in Theorem 4 .

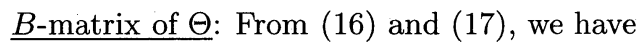

$$
\widehat{D}_{y}^{-T} D_{z}^{T} J_{\gamma} D_{z} \widehat{D}_{y}^{-1}=E_{\Sigma}^{T} J_{p r} E_{\Sigma} \text {. }
$$

Let $E_{z}$ be a solution of $D_{z}^{T} J_{\gamma} D_{z}=E_{z}^{T} J_{p r} E_{z}$. We have $D_{z}^{T} J_{\gamma} D_{z}=\left[\begin{array}{cc}D_{11}^{T} D_{12}^{\dagger T} & I_{r} \\ I_{p} & 0\end{array}\right]\left[\begin{array}{cc}D_{12}^{T} D_{12} & 0 \\ 0 & -R\end{array}\right]\left[\begin{array}{cc}D_{12}^{\dagger} D_{11} & I_{p} \\ I_{r} & 0\end{array}\right]$

where $R:=\gamma^{2} I_{r}-D_{11}^{T}\left(I_{m}-D_{12}\left(D_{12}^{T} D_{12}\right)^{-1} D_{12}^{T}\right) D_{11}$. Since $D_{z}^{T} J_{\gamma} D_{z}$ in (66) has $r$ negative eigenvalues, due to Sylvester's inertia law' $R$ must be positive definite. Let $U \in \Re^{(p \times p)}$ and $V \in \Re^{(r \times r)}$ be nonsingular matrices such that $U^{T} U=D_{12}^{T} D_{12}, V^{T} V=R$, respectively. Equation (66) implies

$$
E_{z}=\left[\begin{array}{cc}
U D_{12}^{\dagger} D_{11} & U \\
V & 0
\end{array}\right]
$$

which is independent of augmentations. Hence, $E_{\Sigma}$ in (65) is given by $E_{\Sigma}=E_{z} \widehat{D}_{y}^{-1}$.

Now, Let us write the transform of the $B$-matrix of $\Theta$ in $(21)$ as

$\left[\begin{array}{c}B^{(u)} \\ B^{(l)}\end{array}\right]:=T^{-1}\left[\begin{array}{c}\left(I-\frac{1}{\gamma^{2}} X Y\right)^{-1}\left(X B_{\Sigma}+C_{\Sigma}^{T} J_{\gamma} D_{\Sigma}\right) \\ W\left(B_{\Sigma}+\frac{1}{\gamma^{2}} Y C_{\Sigma}^{T} J_{\gamma} D_{\Sigma}\right)\end{array}\right] E_{\Sigma}^{-1}$

Frist, we consider $B^{(u)}$ in (68). From (46), we have

$$
\begin{aligned}
& T_{Y}^{-1}\left(I-\frac{1}{\gamma^{2}} X Y\right)^{-1} T_{Y}=\left(I-\frac{1}{\gamma^{2}} T_{Y}^{-1} X T_{Y}^{-T}\left[\begin{array}{cc}
Y_{0} & 0 \\
0 & 0
\end{array}\right]\right)^{-1} \\
& =\left[\begin{array}{cc}
I_{n-\rho_{21}}-\frac{1}{\gamma^{2}} \bar{T}_{Y 1} X \bar{T}_{Y 1}^{-T} Y_{0} & 0 \\
* & I_{\rho_{21}}
\end{array}\right]^{-1},
\end{aligned}
$$


which implies that $T_{Y}^{-1}\left(I-\frac{1}{\gamma^{2}} X Y\right)^{-1} T_{Y}$ has a lower triangular form.

Since the identity (33) holds in the case of the maximum augmentation (23), we have

$$
T_{Y}^{T} Y T_{Y} \cdot T_{Y}^{-1} \widehat{C}^{T}=-T_{Y}^{T}\left(B_{w}+L D_{w} J_{\gamma}^{\prime}\right) \widehat{D}_{w}^{T} .
$$

Using (44) and (46), we can rewrite the relation (70) as

$$
T_{Y 1} \widehat{C}^{T}=-Y_{0}^{-1} T_{Y 1}^{T}\left(B_{w}+L D_{w} J_{\gamma}^{\prime}\right) \widehat{D}_{w}^{T} .
$$

From (28) and (71), it follows that

$$
T_{Y}^{-1} C_{\Sigma}^{T}=\left[\begin{array}{c}
-Y_{0}^{-1} T_{Y 1}^{T}\left(B_{w}+L D_{w} J_{\gamma}^{\prime}\right) \\
*
\end{array}\right]\left[\begin{array}{cc}
0 & -I_{r} \\
I_{m} & 0
\end{array}\right] .
$$

Therefore, (67), (69) and (72) enable us to rewrite the second term of $B^{(u)}$ in (68) as

$$
\begin{aligned}
& T_{Y}^{-1}\left(I-\frac{1}{\gamma^{2}} X Y\right)^{-1} T_{Y} \cdot T_{Y}^{-1} C_{\Sigma}^{T} J_{\gamma} D_{\Sigma} E_{\Sigma}^{-1} \\
&=- T_{Y}^{-1}\left(I-\frac{1}{\gamma^{2}} X Y\right)^{-1} T_{Y 1} \cdot Y_{0}^{-1} T_{Y 1}^{T} . \\
&\left(B_{w}+L D_{w} J_{\gamma}^{\prime}\right)\left[\begin{array}{cc}
0 & -I_{r} \\
I_{m} & 0
\end{array}\right] J_{\gamma} D_{z} E_{z}^{-1} \\
&=-T_{Y}^{-1}\left(I-\frac{1}{\gamma^{2}} X Y\right)^{-1} T_{Y 1} \cdot Y_{0}^{-1} T_{Y 1}^{T} . \\
& {\left[* \gamma^{2}\left(B_{1}+L_{y} D_{21}+L_{z} D_{12} D_{12}^{\dagger} D_{11}\right) V^{-1}\right] . }
\end{aligned}
$$

Based on Theorem 3, we eliminate the unobservable part of $B^{(u)}$ to have

$$
\begin{aligned}
& {\left[I_{n-\rho_{21}} 0\right] B^{(u)}=\bar{T}_{Y 1}\left(I-\frac{1}{\gamma^{2}} X Y\right)^{-1} .} \\
& \quad\left\{X B_{\Sigma}-T_{Y} \cdot T_{Y}^{-1} C_{\Sigma}^{T} J_{\gamma} D_{\Sigma}\right\} E_{\Sigma}^{-1} \\
& =\bar{T}_{Y 1}\left(I-\frac{1}{\gamma^{2}} X Y\right)^{-1} \cdot\left\{X\left[*\left(B_{1}-B_{2} D_{12}^{\dagger} D_{11}\right) V^{-1}\right]\right. \\
& \left.-T_{Y 1} Y_{0}^{-1} T_{Y 1}^{T}\left[* \gamma^{2}\left(B_{1}+L_{y} D_{21}+L_{z} D_{12} D_{12}^{\dagger} D_{11}\right) V^{-1}\right]\right\} \\
& =:\left[* B_{\theta 1}\right] .
\end{aligned}
$$

Next, $B^{(l)}$ in (68) is simplified as follows. Equations (28) and (33) give

$$
\begin{aligned}
& B_{\Sigma}+\frac{1}{\gamma^{2}} Y C_{\Sigma}^{T} J_{\gamma} D_{\Sigma} \\
& =\left\{B-\frac{1}{\gamma^{2}}\left(B_{w}+L D_{w} J_{\gamma}^{\prime}\right)\left[\begin{array}{cc}
0 & -I_{r} \\
I_{m} & 0
\end{array}\right] J_{\gamma} D_{z}\right\} \widehat{D}_{y}^{-1} \\
& =\left(\left[\begin{array}{ll}
0 & B_{2}
\end{array}\right]-L D_{w}\left[\begin{array}{cc}
0 & -I_{r} \\
I_{m} & 0
\end{array}\right] D_{z}\right) \widehat{D}_{y}^{-1} .
\end{aligned}
$$

Here, we used the identities

$B_{w}\left[\begin{array}{cc}0 & -I_{r} \\ I_{m} & 0\end{array}\right] J_{\gamma}=\gamma^{2}\left[\begin{array}{ll}B_{1} & 0\end{array}\right], J_{\gamma}^{\prime}\left[\begin{array}{cc}0 & -I_{r} \\ I_{m} & 0\end{array}\right] J_{\gamma}=\gamma^{2}\left[\begin{array}{cc}0 & I_{m} \\ -I_{r} & 0\end{array}\right]$.

Equations (67) and (75) imply that $B^{(l)}$ in (68) is given by

$$
\begin{aligned}
& {\left[I_{n-\rho_{12}} 0\right] B^{(l)}=\bar{T}_{X 1} W\left(\left[\begin{array}{ll}
0 & B_{2}
\end{array}\right]-L D_{w}\left[\begin{array}{cc}
0 & -I_{r} \\
I_{m} & 0
\end{array}\right] D_{z}\right) E_{z}^{-1}} \\
& =\bar{T}_{X 1} W\left[*-\left\{\left(B_{2}+L_{z} D_{12}\right) D_{12}^{\dagger} D_{11}+L_{y} D_{21}\right\} V^{-1}\right] \\
& =:\left[* B_{\theta 2}\right]
\end{aligned}
$$

$\underline{C \text {-matrix of } \Theta}$ : From (72), it follows that

$$
\begin{aligned}
& -\frac{1}{\gamma^{2}} C_{\Sigma} Y T_{Y}=-\frac{1}{\gamma^{2}} C_{\Sigma} T_{Y}^{-T}\left[\begin{array}{cc}
Y_{0} & 0 \\
0 & 0
\end{array}\right] \\
& =\left[\frac{1}{\gamma^{2}}\left[\begin{array}{cc}
0 & I_{m} \\
-I_{r} & 0
\end{array}\right]\left(B_{w}+L D_{w} J_{\gamma}^{\prime}\right)^{T} T_{Y 1} 0\right] .
\end{aligned}
$$

Routine calculations using the definition of $B_{w}, D_{w}$ and $L$ yield

$$
\begin{aligned}
& {\left[\begin{array}{ll}
C_{\theta 11} & 0 \\
C_{\theta 21} & 0
\end{array}\right]:=-\frac{1}{\gamma^{2}} C_{\Sigma} Y T_{Y}^{-T}} \\
& =\left[\left[\begin{array}{c}
-L_{z}^{T} \\
-\frac{1}{\gamma^{2}}\left(B_{1}+L_{z} D_{11}+L_{y} D_{21}\right)^{T}
\end{array}\right] T_{Y 1} \quad 0\right] .
\end{aligned}
$$

Furthermore, from (54), we have

$\left[\begin{array}{ll}C_{\theta 12} & 0 \\ C_{\theta 22} & 0\end{array}\right]:=\left(C_{\Sigma}+D_{\Sigma} F_{\Sigma}\right) T_{X}=\left[\left(C_{z}+D_{z} F\right) T_{X 1} 0\right]$.

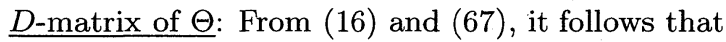

$$
\begin{gathered}
D_{\Sigma} E_{\Sigma}^{-1}=D_{z} E_{z}^{-1}=\left[\begin{array}{cc}
D_{11} & D_{12} \\
I & 0
\end{array}\right]\left[\begin{array}{cc}
0 & V^{-1} \\
U^{-1} & -D_{12}^{\dagger} D_{11} V^{-1}
\end{array}\right] \\
=\left[\begin{array}{c}
*\left(I-D_{12} D_{12}^{\dagger}\right) D_{11} V^{-1} \\
0
\end{array}\right]:=\left[\begin{array}{ll}
* & D_{\theta 1} \\
0 & D_{\theta 2}
\end{array}\right] .
\end{gathered}
$$

[Step 2] Now, to derive the closed-loop transfer function $\Phi$, we describe the $\left\{2 n-\left(\rho_{12}+\rho_{21}\right)\right\}$-th order $J$-lossless system. Using (64), (74), (76), (78), (79) and (80), we can rewrite (21) as

$$
\Theta=\left[\begin{array}{ll}
\Theta_{11} & \Theta_{12} \\
\Theta_{21} & \Theta_{22}
\end{array}\right]=\left[\begin{array}{cc|cc}
A_{\theta 1} & 0 & * & B_{\theta 1} \\
0 & A_{\theta 2} & * & B_{\theta 2} \\
\hline C_{\theta 11} & C_{\theta 12} & * & D_{\theta 1} \\
C_{\theta 21} & C_{\theta 22} & * & D_{\theta 2}
\end{array}\right] .
$$

[Step 3] Therefore, the closed-loop transfer function $\Phi(s)$ for the central $H^{\infty}$ controller is given by

$$
\begin{aligned}
& \Phi(s)=\Theta_{12} \Theta_{22}^{-1} \\
& =\left[\begin{array}{cc|c}
A_{\theta 1} & 0 & B_{\theta 1} \\
0 & A_{\theta 2} & B_{\theta 2} \\
\hline C_{\theta 11} & C_{\theta 12} & D_{\theta 1}
\end{array}\right]\left[\begin{array}{cc|c}
A_{\theta 1} & 0 & B_{\theta 1} \\
0 & A_{\theta 2} & B_{\theta 2} \\
\hline C_{\theta 21} & C_{\theta 22} & D_{\theta 2}
\end{array}\right]^{-1}
\end{aligned}
$$

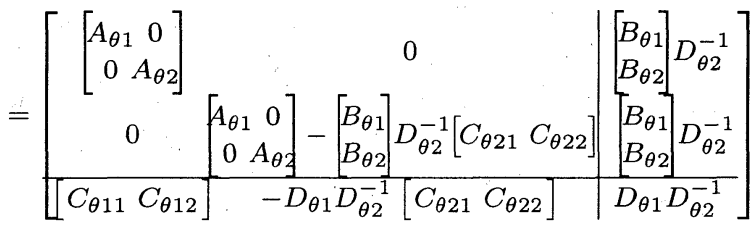

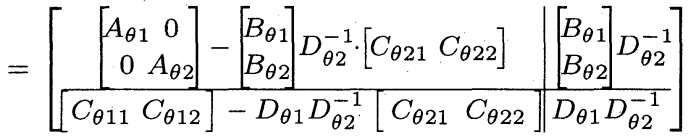

$$
\begin{aligned}
& =:\left[\begin{array}{c|c}
A_{c l} & B_{c l} \\
\hline C_{c l} & D_{c l}
\end{array}\right],
\end{aligned}
$$

where the similarity transformation

$$
T_{\Phi}=\left[\begin{array}{cc}
I_{2 n-\left(\rho_{12}+\rho_{21}\right)} & I_{2 n-\left(\rho_{12}+\rho_{21}\right)} \\
0 & I_{2 n-\left(\rho_{12}+\rho_{21}\right)}
\end{array}\right]
$$

is used. Lengthy but straightforward calculations for (82) and (83) yield the representations of $A_{c l}, B_{c l}, C_{c l}$ and $D_{c l}$ in $(61)$.

\section{Conclusion}

We have investigated the McMillan degree of closedloop transfer function of $H^{\infty}$ control systems. Our result 
implies that the intrinsic complexity of closed-loop transfer function is determined by the number of the stable invariant zeros of $P_{12}(s)$ and $P_{21}(s)$. Based on this result, we have obtained an explicit form of the $\left\{2 n-\left(\rho_{12}+\rho_{21}\right)\right\}$ th order closed-loop transfer function of $H^{\infty}$ control systems. We have also provided a new interpretation of the notion of the maximum augmentation. Our results based on the chain-scattering approach provide clear insights into the closed-loop structure of $H^{\infty}$ control systems.

Acknowledgement: This work is supported in part by Grant-in-Aid for JSPS Fellows from The Ministry of Education, Science, Sports and Culture of Japan.

\section{References}

1) B. A. Francis, J. W. Helton and G. Zames: $H^{\infty}$-optimal feedback controllers for linear multivariable systems, IEEE Trans. Automatic Control, 29, 888/900 (1984)

2) K. Glover: All optimal Hankel-norm approximations of linear multivariable systems and their $L^{\infty}$-error bounds, International Journal of Control, 39, 1115/1193 (1984)

3) H. Kimura: On interpolation-minimization problem in $H^{\infty}$, Control Theory and Advanced Technology, 2, 1/25 (1986)

4) K. Glover and J. C. Doyle: State-space formulae for all stabilizing controllers that satisfy a $H^{\infty}$ norm bound and relations to risk sensitivity, Systems \& Control Letters, 11, $167 / 172(1988)$

5) S. Hara, T. Sugie and R. Kondo: $H^{\infty}$ control problem with $j \omega$-axis zeros, Automatica, 28, 55/70 (1992)

6) K. Z. Liu and T. Mita: Generalized $H^{\infty}$ control theory, Proceedings of American Control Conference, 2245/2249 (1992)

7) X. Xin, T. Mita and H. Kimura: $\left(J, J^{\prime}\right)$-lossless factorization approach to $H^{\infty}$ control problems with infinite and finite $j \omega$-axis zeros, Proceedings of the 38 th Conference on Decision and Control, Phoenix (1999)

8) W. Kongprawechnon and II. Kimura: J-lossless factorization and $H^{\infty}$ control for discrete-time systems, International Journal of Control, 65, 867/884 (1996)

9) L. Baramov and H. Kimura: Nonlinear $L_{2}$-gain suboptimal controllers, Automatica, 33, 1247/1262 (1997)

10) P. H. Lee, H. Kimura and Y. C. Soh: Chain-scattering approach to $H^{\infty}$ control for time-varying systems, International Journal of Control, 71, 195/218 (1998)

11) H. Kimura: Chain-Scattering Approach to $\mathrm{H}^{\infty}$-Control, Birkhäuser (1996)

12) R. E. Kalman: Contributions to the theory of optimal control, Bol. Soc. Matem. Mex., 102/119 (1960)

13) M. A. Athans: The role and use of the stochastic Linear-Quadratic-Gaussian problem, IEEE Trans. Automatic Control, 16, 529/552 (1971)

14) D. G. Luenberger: An introduction of observers, IEEE Trans. Automatic Control, 16, 596/602 (1971)

15) M. Green and D. J. N. Limebeer: Linear Robust Control, Prentice Hall (1995)

16) D. J. N. Limebeer and Y. S. Hung: An analysis of the pole-zero cancellations in $H^{\infty}$-optimal control problem of the first kind, SIAM J. Control and Optimization, 25, 6, 1457/1493 (1987)

17) D. J. N. Limebeer: An interpolation theory approach to $H^{\infty}$ controller degree bounds, Linear Algebra and its Applications, 98, 347/386 (1988)
18) S. Ushida, T. Ohba and H. Kimura: Closed-loop structure of $H^{\infty}$ control, Proceedings of the 38th Conference on Decision and Control, 3150/3155, Phoenix (1999)

19) J. C. Doyle, K. Glover, P. P. Khargonekar and B. A. Francis, "State-space solutions to standard $H_{2}$ and $H_{\infty}$ control problems, IEEE Trans. Automatic Control, 26, 4/16 (1989)

20) K. Zhou, J. C. Doyle and K. Glover: Robust and Optimal Control, Prentice Hall (1995)

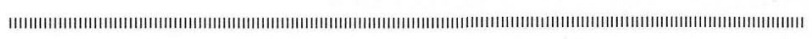
Shun Ushid A (Student Member)

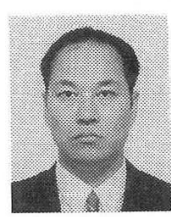

He received B. S. and M. S. degrees from Osaka University in 1995 and 1997, respectively. $\mathrm{He}$ is currently a $\mathrm{Ph}$. D. student of the University of Tokyo. He has been a JSPS Research Fellow since 1997-2000. He is a member of SICE and ISCIE. His research interests include robust control theory and modeling.

\section{Toshitomo Онва (Student Member)}

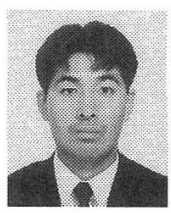

He received B. S. and M. S. degrees from the University of Tokyo in 1997 and 1999, respectively. He is currently a $\mathrm{Ph}$. D. student of the University of Tokyo. He is a member of SICE. His research interests include robust control theory.

\section{Hidenori Kimura (Member)}

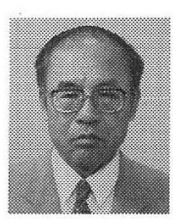

He received the degree of the Doctor of Engineering from the University of Tokyo in 1970. He jointed the Faculty of Engineering Science, Osaka University where he engaged in research and education of control theory and its applications, signal processing and system theory for 25 years. In 1974-1975, he stayed in Imperial College of Science and Technology and Warick University in UK supported by the British Council. In 1995, he moved to the Faculty of Engineering, the University of Tokyo. He is now interested in robust control, learning theory and modeling. He received the paper award and author's award from SICE several times. He was also a recipient of the George Axelby Paper Award from IEEE CSS in 1984 and the Paper Prize Award from IFAC in 1984 and 1990. He is a Fellow of SICE and IEEE.

\section{Yasuaki Oısнi (Member)}

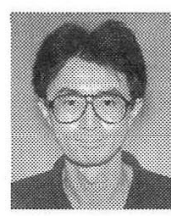

He received the Bachelor, Master, and Doctor of Engineering degrees from the University of Tokyo in 1990, 1993, and 1998, respectively. Since 1995, he has been with Department of Mathematical Engineering and Information Physics, the University of Tokyo, as a Research Associate. His research interests include system identification, learning theory, robust control and sampled-data control. 